\title{
COMMUNICATIONS
}

\section{Design of microwave lowpass filter based on log periodic zigzag DGS}

\author{
Rong-Bin Chen ${ }^{1}$, Xiao-Ou Ou ${ }^{2}$, Jia-Lin $\mathbf{L i}^{3 *}$, \\ Baidenger Agyekum Twumasi ${ }^{3,4}$
}

\begin{abstract}
This contribution presents, for the first time, the design of microwave lowpass filter with ultrawide stopband and low insertion loss based on the log-periodic zig-zag defected ground structure (DGS); the design is specifically evolved from the principle of the log-periodic line antenna and the slotline based DGS. Based on the log-periodic property, studies show that ultra-wideband suppression can be realized by increasing the flare angle or the angle subtended by each element of the slotline DGS. The developed filter therefore shows low insertion loss of $0.7 \mathrm{~dB}$ within the passband, sharp transition between the passband and the stopband and ultra-wide suppression band from 2.61-26.5 GHz with a $20 \mathrm{~dB}$ suppression level. The DGS architecture is simple and easy to realize, and the design is thus useful in many microwave applications. The fabricated prototype filter validates the study, both from simulations and from measurements.
\end{abstract}

K e y w or d s: log periodic, zigzag, slotline, defected ground structure (DGS), microwave filter

\section{Introduction}

In today's wireless communication and the increasing connectivity of things (internet of things), microwave filters and other related components are indispensable currently and in the nearfuture [1]-[4]. Compact size and high-performance low profile microstrip lowpass filters (LPFs) with wide stopband, good return loss and low insertion loss are in high demand for various wireless applications. This is due to the ability of such filters to suppress wideband noise and spurious signals. Also due to easy fabrication and the lower cost of microstrip based filters, it reduces cost whiles enhancing system performance. Many microstrip based filters have been produced till date, but it is important that the performance of such filters needs to be further improved for all related applications. Hence the continuous performance improvement of such filters being experienced including this contribution. LPFs are vital components that have found wide applications in todays wireless communication system for noise and intermodulation signals suppression.

In [5], complementary split ring resonators were used to design compact low insertion loss LPF with sharp transition between the passband and the stopband. In the design, the lowpass band insertion loss was good, but the suppression band is not wide enough. In [6], an accurate model of a rectangular bracket DGS was presented and further validated by cascaded two and three DGS cells. In [7], the hairpin shaped DGS was studied and further developed for microwave filter applications. The developed demonstrator achieved a compact size and wide stopband suppression. In [8], Yagi shaped DGS was also exploited for microwave filter applications and demonstrated good performance. In [9], a miniaturized LPF with improved Koch Fractal DGS was proposed with sharp transition and ultra-wideband stopband as compared to the traditional dumbbell-shaped DGS filters. In [10], a compact microstrip LPF has been proposed with sharp roll-off and a wide stopband. It also characterized a high figure of merit with a compact size.

In general, LPFs with ultra-wideband suppression and sharp roll-off as well as low insertion loss within the passband are very much desired for many practical applications. In this contribution, a log-periodic zigzag slotline based DGS is reported, for the first time, for microstrip LPF applications. The developed filter features ultrawide stopband and low insertion loss within the passband as well as sharp transition between the passband and the stopband. To validate the study, a prototype filter is fabricated and confirmed, both from simulations and measurements.

\section{Log periodic zigzag DGS}

The proposed DGS is based on the principles of the $\log$ periodic antenna design [11][15] and the slotline based technique. These slots are etched on the ground plane of a microwave substrate under a microstrip line. It is known that the log periodic antenna is a frequency independent antenna composed of resonant elements. It can achieve frequency independence by having its resonant elements staggered so that as frequency is varied, thus the function

\footnotetext{
${ }^{1}$ Department of Electronic and Information Technology, Jiangmen Polytechnic, Jiangmen 529030, China, ${ }^{2}$ Jiangmen Branch, China Mobile Group Guangdong Co., Ltd., Jiangmen 529000, China, ${ }^{3}$ School of Physics, University of Electronic Science and Technology of China, Chengdu 611731, China, ${ }^{4}$ Electrical /Electronic Engineering Department, Ho Technical University, Ho, Ghana, chenrongbin@139.com, ouxiaoou2@gd.chinamobile.com, jialinli@uestc.edu.cn, btwumasi@htu.edu.gh
} 
of the resonant element is transferred smoothly from one element to the next. For an antenna array made up of similar discrete elements, this implies that the physical dimensions of the elements should be scaled from one to the next such that the required frequency range is covered with elements having overlapping response [11]. In [12], a log periodic-Yagi-Uda array was studied where the principle of the log periodic was applied to the Yagi-Uda technique to realize the bandwidth enhancement of the basic Yagi-Uda antenna. The antenna in [12] was made of individual cells each resembling the basic Yagi-Uda but each cell has different size by a geometric constant, where the driven element was fed by a common transmission line. This shows the versatility in application of the log-periodic structure and, in principle, to various microwave applications. In view of such type of application, this contribution proposes a DGS structure based on the log-periodic array to develop the microwave lowpass filter. For the general case of the log periodic antenna, the resonant elements are parallel to each other but for this proposed log-periodic DGS, the resonant elements etched on the ground plane have been arranged in a zig-zag fashion (slanted resonant elements) with some small gap between the tip of each vertex of the zig-zag elements, as shown in Fig. 1. Such a fashion constitutes a slot array under a certain periodicity.

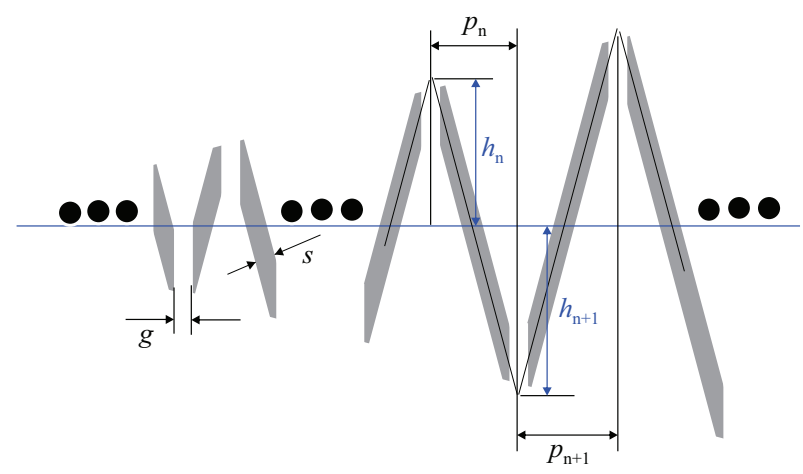

Fig. 1. The proposed slotline based log periodic zigzag DGS

It is shown in Fig. 1 that referred to the horizontal axis (corresponding to the central line, which is also the direction of placed microstrip line), the height from the vertex of each zig-zag to the central line is $h_{n}$ for the $n^{\text {th }}$ slanted slot, where $\mathrm{n}$ takes on all positive or negative integral values. Similarly, it is marked as $h_{n+1}$ for the $(n+1)^{\text {th }}$ slot. The spacing between two adjacent vertexes is $p_{n}$ related to the $n^{\text {th }}$ slot. These two parameters are of the geometric ratio or called scale factor that is generally given by: for the height ratio

$$
\frac{1}{\tau_{h}}=\frac{h_{n+1}}{h_{n}},
$$

and for the spacing ratio

$$
\frac{1}{\tau_{p}}=\frac{p_{n+1}}{p_{n}},
$$

where both ratios are less than 1 .

To separate the slots between two adjacent vertexes of the zigzag, a small gap that is marked as $\mathrm{g}$ is introduced at the tip of each vertex as shown in Fig. 1. Notice that if carried to the limit, the array would converge to the point 0 on the left when $n$ approaches $-\infty$, and becomes infinite in extent on the right as $n$ arrives $\infty$.

For such a kind of DGS, the height $h_{n}$ and spacing $p_{n}$ co-determine the length of the related slot and further determine its resonance. On the other hand, to be useful as a practical application, it must be possible to truncate the slot array so that at all frequencies above some lower limit, the truncated array performs like the infinite structure. The lower resonance is thus determined by the longest slot while the shortest one determines the upper resonance. The last parameter is related to the characteristic impedance of the slot that is marked as slot width $s$ in Fig. 1. This characteristic impedance could influence the impedance matching of the microstrip transmission line placed above the proposed DGS structure in this study.

\section{Developing microstrip filter based on the proposed DGS}

To demonstrate the potential application of the proposed DGS, a microstrip LPF is developed here. The filter is designed on a microwave substrate having a relative permittivity of 9.6 and a thickness of $0.8 \mathrm{~mm}$. The dedicated filter has a cut-off frequency of $f_{c}=2 \mathrm{GHz}$ and the expected suppression band is up to $24 \mathrm{GHz}$, namely $12 f_{c}$, thus a very wideband suppression. On the abovementioned substrate, the longest slot should have a length of approximately $24 \mathrm{~mm}$ when the slot functions as a half-wavelength resonator at $f_{c}$. Correspondingly, in this study, we set its height $h_{n}$ to be $16 \mathrm{~mm}$. Meanwhile, we also set $\tau_{h}=\tau_{p}=\tau$ to simplify the design. The rest parameter could be the initial periodicity that would be marked as $p_{n-1}$ based on Fig. 1 . The scale factor $\tau$ is sweeping studied here and referred to the related studies, it is sweeping from 0.7 to 0.9 in this work. The shortest slot, of course, determined by the upper suppression margin, can thus be determined by the scale factor $\tau$ and the initial height $h_{n}$ as well as the initial periodicity $p_{n-1}$ under a certain periodicity number $n$. The longest and shortest slots are, as mentioned before, formulating the truncation for such an infinite slot array. The microstrip line used in this study is a standard $50 \Omega$ transmission line placed on the substrate above the proposed DGS structure. Now considering that, the initial slot is serving as a half-wavelength resonator with a fundamental resonance of $2 \mathrm{GHz}$, and the initial height $h_{n}=16 \mathrm{~mm}$ as well as a sweeping scale factor $\tau=0.7-0.9$, the initial periodicity $p_{n-1}$ is set as $8 \mathrm{~mm}$ and sweeping varied from $7 \mathrm{~mm}$ to $9 \mathrm{~mm}$. With these initial values, the studied filter can be 


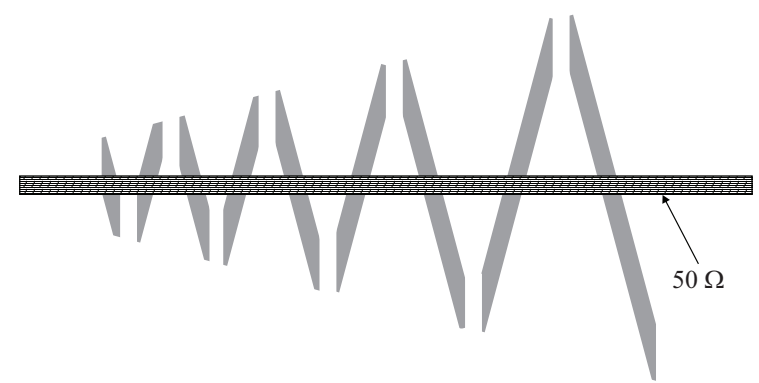

Fig. 2. Layout of the proposed log periodic zigzag DGS prototype filterwhere the gray section denotes the patterned ground while the shaded section represents the microstrip transmission line
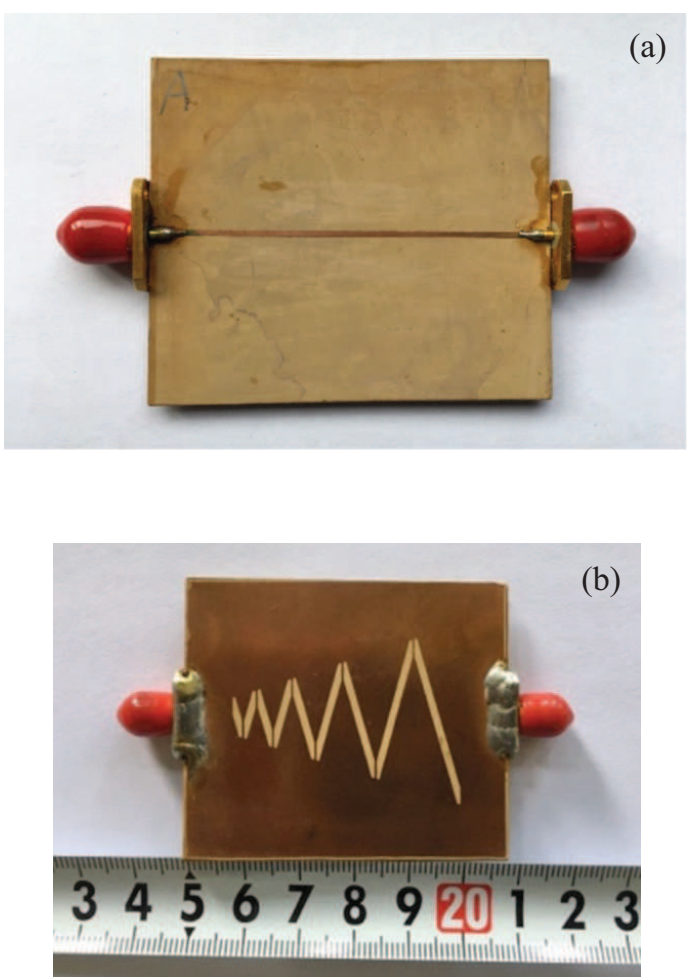

Fig. 3. Photograph of the fabricated logperiodic DGS prototype filterafront sidebback side

developed with the use of full-wave electromagnetic (EM) simulations, here is from Ansoft Ensemble 8.0. Figure 2 shows the layout of the studied prototype filter.

After sweeping studies and full-wave EM optimizations, the dedicated filter is developed under a suppression level of $20 \mathrm{~dB}$ in the suppression band and an in-band return loss of $10 \mathrm{~dB}$. Results show a total of 10 sections (namely the periodicity number is $n=10$ ) of slots are employed. Hence $h_{10}=16 \mathrm{~mm}$, while the remaining optimized parameters are $\tau=0.8352$, and $p_{9}=8.4414 \mathrm{~mm}$. Thus the first slot has a height of $h_{1}=16 \times 0.8352^{9}=$ $3.164 \mathrm{~mm}$, and $p_{1}=8.4414 \times 0.8352^{8}=1.999 \mathrm{~mm}$. The slot has a width of $s=1.213 \mathrm{~mm}$ while the small gap at the vertex of each two slots is fixed as $g=0.45 \mathrm{~mm}$ in this study. The occupied area of this demonstrator is found to be $40.65 \times 29.546 \mathrm{~mm}^{2}$.

\section{Experimental characterization of the developed filter}

The developed prototype filter was fabricated and experimentally characterized for the electric performance. Figure 3 shows the photograph of the developed filter. The measurement was carried out by a vector network analyzer (VNA) from Agilent technologies under a model number N9918A with full two-port calibrations.
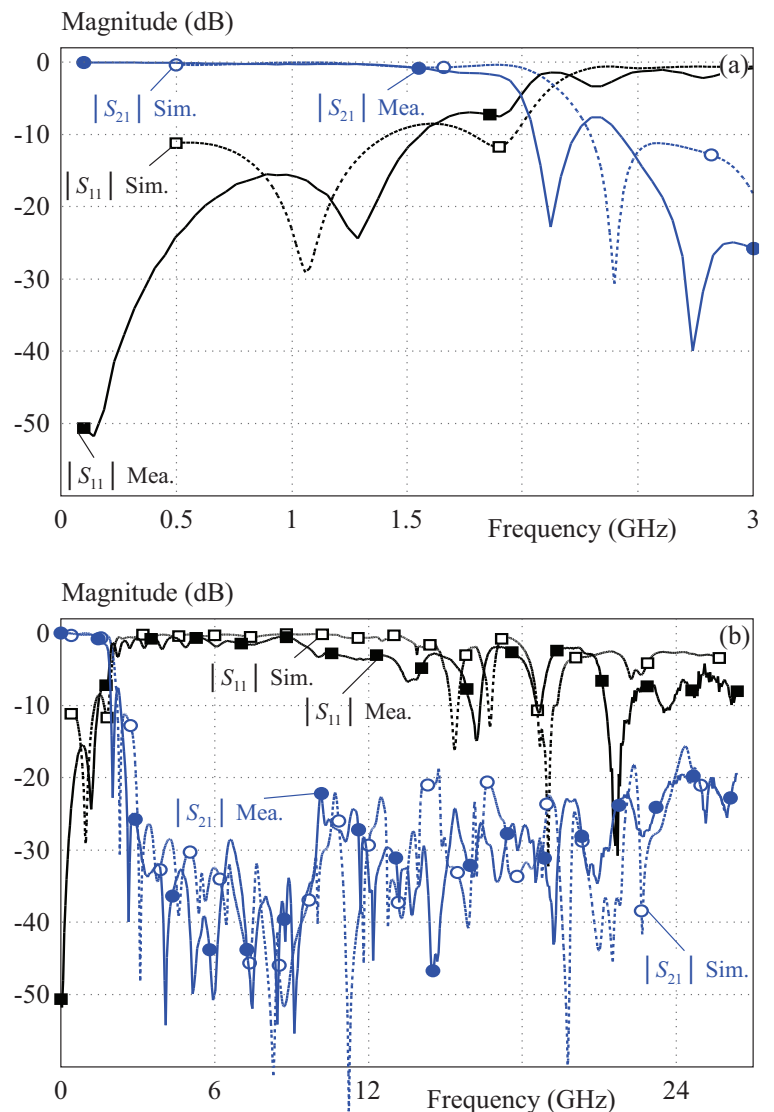

Fig. 4. Simulated and measured electric performance of the proposed log periodic DGS prototype filterainband responsesbwideband performance

Figure 4 shows the simulated and measured results for the fabricated prototype filter. It is found from measurements that the prototype filter has a cut-off frequency of approximately $1.96 \mathrm{GHz}$ and for comparisons, the simulated response is also presented. The simulated cut-off frequency is about $2.14 \mathrm{GHz}$, as described in Fig. 4(a) for the in-band response. From DC to about $1.85 \mathrm{GHz}$, the insertion loss is better than $1 \mathrm{~dB}$ from measurements especially, it is less than $0.7 \mathrm{~dB}$ from DC to $1.54 \mathrm{GHz}$. Notice that the loss includes the conductor loss of the transmission line and that of the two SMA connectors at the two ends as well as the transitions between the connectors and the microstrip line. The measured in-band 
Table 1. Comparisons of some related DGS filters and this study

\begin{tabular}{|c|c|c|c|c|c|c|c|}
\hline Refs./year & $\begin{array}{c}f_{c} \\
(\mathrm{GHz})\end{array}$ & $\begin{array}{c}\text { Passband } \\
\text { insertion } \\
\text { loss } \\
\text { (dB) }\end{array}$ & $\begin{array}{c}\text { Stopband } \\
\text { range }(\mathrm{GHz}) \\
\text { /suppression } \\
\text { level }(\mathrm{dB})\end{array}$ & $\begin{array}{l}\text { Filter size } \\
\left(\mathrm{mm}^{2}\right)\end{array}$ & \multicolumn{2}{|r|}{$\begin{array}{c}\text { DGS } \\
\text { pattern }\end{array}$} & Notes \\
\hline$(16) / 2016$ & 3.88 & 0.3 & $4.07-15 / 20$ & $35.25 \times 16.15$ & \multicolumn{2}{|c|}{ Dumbbell } & Open stubs, spur-line, \\
\hline$(16) / 2016$ & 3.88 & 0.3 & $4.07-15 / 20$ & $35.25 \times 16.15$ & \multicolumn{2}{|c|}{ Dumbbell } & defected microstrip line \\
\hline$(17) / 2017$ & 1.1 & 0.3 & $1.3-8.9 / 20$ & $59.2 \times 35.52$ & \multicolumn{2}{|c|}{ Dumbbell } & Defected microstrip line, \\
\hline$(17) / 2017$ & 1.1 & 0.3 & $1.3-8.9 / 20$ & $59.2 \times 35.52$ & \multicolumn{2}{|c|}{ Dumbbell } & compensated capacitor* \\
\hline$(18) / 2018$ & 2.4 & 0.35 & $2.8-6 / 20$ & $20.86 \times 11.92$ & \multicolumn{2}{|r|}{ Modified dumbbell } & Split ring resonator \\
\hline$(19) / 2019$ & 2.2 & 1.9 & $2.7-12 / 50$ & $100 \times 2.6$ & \multicolumn{2}{|r|}{ Pi-shaped } & Standard microstrip \\
\hline$(20) / 2020$ & 2.11 & / & $3-20 / 18$ & $15.5 \times 12.7$ & \multicolumn{2}{|c|}{ Meandered line } & \\
\hline$(20) / 2020$ & 2.11 & / & $3-20 / 18$ & $15.5 \times 12.7$ & \multicolumn{2}{|c|}{ loaded square } & Spur line \\
\hline$(21) / 2020$ & 6 & 1.1 & / & $42 \times 20$ & \multicolumn{2}{|c|}{ circular } & Standard \\
\hline This work & 1.96 & 0.7 & $2.61-26.5 / 20$ & $40.65 \times 29.546$ & \multicolumn{2}{|r|}{ Log periodic zigzag } & microstrip \\
\hline & & Refs. & (17) & (19) & $(20)$ & This $\mathrm{w}$ & \\
\hline & & $\varepsilon_{\mathrm{r}}$ & 3.38 & 3.8 & 4.4 & 10.2 & \\
\hline & & $h(\mathrm{~mm})$ & 0.813 & 0.254 & 0.8 & 0.635 & \\
\hline
\end{tabular}

group delay is $0.4 \mathrm{~ns}$ in general. Meanwhile, both simulation and measurement indicate that, the proposed filter has a sharp transition from the passband to the stopband.

The wideband suppression response is recorded in Fig. 4(b). It is measured from $2.61 \mathrm{GHz}$ to over $26.5 \mathrm{GHz}$, a high and wideband suppression is found under a suppression level of $20 \mathrm{~dB}$.

Further, for performance comparisons, Tab. 1 presents the performance of the proposed filter and some reported works. it can be observed that the proposed filter has comparable good performance and an ultra-wide suppression band with a compact size. It should be noted that this is the first time to study and develop the log periodic zigzag fashion for potential applications to the microwave filters.

\section{Conclusion}

For the first time, the log-periodic zig-zag DGS has been exploited and proposed for microwave filter applications. Developing the proposed microwave filter is based on the design principles of the log-periodic antenna. The studied filter has been fabricated and experimentally characterized with promising performance, including low insertion loss in the passband and ultra-wide suppression band beyond the passband as well as sharp transition from the passband to the stopband. With these performances, the proposed filter topology is believed to be good potential for further integration with system applications.

\section{REFERENCES}

[1] J.-L. Li and B.-G. Yu, "Numerical study and experimental validation of a four-way feeding network with $45 \mathrm{deg}$ broadband phase shifts for antenna array applications", Journal of Electrical Engineering, vol. 70, no. 5, pp. 400-405, 2019.

[2] L. Xia, J.-L. Li, Z. Ji, and S.-S. Gao, "An in-line coaxial-to-circular waveguide transition at X band", Journal of Electrical Engineering, vol. 71, no. 1, pp. 55-59, 2020.

[3] C.-G. Sun, J.-L. Li, and B.-A. Twumasi, "Multi-way differential power divider with microstrip output interfaces", Journal of Electrical Engineering, vol. 71, no. 4, pp. 274-280, 2020.

[4] J.-L. Li, J. X. Chen, Q. Xue, J.-P. Wang, W. Shao, and L.-J. Xue, "Compact microstrip lowpass filter based on defected ground structure and compensated microstrip line", IEEE MTT-S International Microwave Symposium Digest, vol. 71, no. 4, pp. 472-14831486.

[5] M.-K. Mandal, P. Mondal, S. Sanyal, and A. Chakrabarty, "Low insertion-loss, sharp-rejection and compact microstrip low-pass filters", IEEE Microwave and Wireless Component Letters, vol. 16, no. 11, pp. 600-602, 2006.

[6] B.-A Twumasi and J.-L. Li, "An equivalent circuit model of a rectangular bracket shaped DGS and its microwave filter applications", Journal of Electrical Engineering, vol. 70, no. 1, pp. 32-38, 2019.

[7] R.-B. Chen and X.-O. Ou, "A hairpin DGS resonator for application to microstrip lowpass filters", Journal of Electrical Engineering, vol. 71, no. 2, pp. 110-115, 2020.

[8] J. Gong, "A rectangular slot Yagi DGS microstrip filter with sharp rolloff and wide suppression band", Journal of Electrical Engineering, vol. 71, no. 3, pp. 217-221, 2020.

[9] Z.-L. Wen, Y.-Y. Han, X.-Y. Sun, and R. Tao, "Design of miniaturized low-pass filter with improved Koch fractal DGS", Proceedings of IEEE International Symposium Electromagnetic Compatibility, pp. 1-4, 2017.

[10] J.-L. Li, S.-W. Qu, and Q. Xue, "Compact microstrip lowpass filter with sharp roll-off and wide stop-band", Electronics Letters, vol. 45, no. 2, pp. 110-111, 2009.

[11] D.-E. Isbell, "Log periodic dipole arrays", IRE Transactions on Antennas and Propagation, vol. 8, no. 3, pp. 260-267, 1960. 
[12] N. Barbano, "Log periodic Yagi-Uda array", IEEE Transactions on Antennas and Propagation, vol. 14, no. 2, pp. 235-238, 1966.

[13] D. Varon and R. Kieburtz, "Theoretical analysis of a log-periodic structure", Proceedings of IEEE International Symposium on Antennas and Propagation, pp. 58-61, 1964.

[14] C.-C. Bantin and K.-G. Balmain, "Study of compressed log-periodic dipole antennas", IEEE Transactions on Antennas and Propagation, vol. 18, no. 2, pp. 195-203, 1970.

[15] R. Lehmensiek and D.-I. De Villiers, "Optimization of log-periodic dipole array antennas for wideband omnidirectional radiation", IEEE Transactions on Antennas and Propagation, vol. 63, no. 8, pp. 3714-3718, 2015.

[16] P. Zhang and M. Li, "A novel sharp roll-off microstrip lowpass filter with improved stopband and compact size using dual-plane structure", Microwave and Optical Technology Letters, vol. 58, no. 5, pp. 1085-1088, 2016.

[17] A. Boutejdar, A.-A. Ibrahim, and R.-M. Shubair, "A novel highperformance DMS/DGS low-pass filter for radar applications", Proceedings on IEEE International Symposium on Antennas Propagation \& USNC/URSI National Radio Science Meeting, pp. 2259-60, 2017.

[18] S. Sen, T. Moyra, and D. Sarkar, "Modelling and validation of microwave LPF using modified rectangular split ring resonators (SRR) and defected structures", AEU-International Journal of Electronics and Communications, vol. 88, pp. 1-10, 2018.

[19] Y. Han, Z. Liu, C. Zhang, C. Mei, Q. Chen, and S. Yuan, "A flexible microstrip low-pass filter design using asymmetric Pi-shaped DGS", IEEE Access, vol. 7, pp. 49999-50006, 2019.

[20] T.-K. Rekha, P. Abdulla, P.-M. Jasmine, and A.-A. Anu, "Compact microstrip lowpass filter with high harmonics suppression using defected structures", AEU-International Journal of Electronics and Communications, vol. 115, pp. 153032-1-10, 2020.

[21] C. Maximo-Gutierrez, J. Hinojosa, F.-L. Martinez-Viviente, and A. Alvarez-Melcon, "Design of high-performance microstrip and coplanar low-pass filters based on electromagnetic bandgap (EBG) structures", AEU-International Journal of Electronics and Communications, vol. 123, pp. 153311-1-7, 2020.

Received 24 October 2020

Rong-Bin Chen was born in January 1980 at Guangdong, China. He received BS Degree from the Shantou University, Shantou, China, in 2003, and MS Degree from the Sun Yat-sen University, Guangzhou, China, in 2005. From July 2005 to June 2018, he was with the Jiangmen Branch of China Mobile Group Guangdong Co., Ltd., Jiangmen, China, where he served as a Technique Supporter for maintenance, construction and development of $2 \mathrm{G} / 3 \mathrm{G} / 4 \mathrm{G} / 5 \mathrm{G}$ wireless/mobile networks. Since June 2018, he has been with the Department of Electronic and Information Technology, Jiangmen Polytechnic, Jiangmen, China. His research interests include wireless communication technology, internet of things.

Xiao-Ou Ou was born in March 1981 at GuangXi, China. She received BS Degree from the Shantou University, Shantou, China, in 2003, and MS Degree from the Sun Yat-sen University, Guangzhou, China, in 2006. From July 2006 to now, she was with the Jiangmen Branch of China Mobile Group Guangdong Co., Ltd., where she served as a Technique Supporter for business. Her research interests include Communication technology, information technology.

Jia-Lin Li was born in October 1972 at Sichuan, China. He received the MS degree from the University of Electronic Science and Technology of China (UESTC), Chengdu, China, in 2004, and the $\mathrm{PhD}$ degree from the City University of Hong Kong, Hong Kong, in 2009, both in electronic engineering. From September 2005 to August 2006, he was a Research Associate with the Wireless Communication Research Center, City University of Hong Kong, Hong Kong. Since September 2009, he has been with the School of Physics, UESTC, where he is currently a Professor. His research interests include microwave/millimeter-wave antenna and arrays, circuits and systems, interactions between microwave and complex medium.

Baidenger Agyekum Twumasi was born in September 1981 at Nsawam in the Eastern Region of Ghana. He obtained the HND in Elect/Electronic Engineering from Ho Polytechnic in 2004, the Master of Telecommunication Management from HAN University of Applied Sciences in Netherlands in 2011, and the PhD of the Electronic Science and Technology from the University of Electronic Science and Technology of China (UESTC) in 2020. He is currently pursing the PostDoc research in the School of Physics, UESTC.. He is currently a PhD candidate in the University of Electronic Science and Technology of China, majoring in Electronic Science and Technology in the School of Physics. He is a lecturer in Elect/Electronic Engineering at Ho Technical University in Ghana. He is also a graduate student member of IEEE and a member of APS and MTTS societies. His research interest includes MIMO Antenna Designs, Planar electronic circuits for Telecommunication Applications, Study of Electromagnetic Time Reversal (EMTR) and its Applications, Wireless Power Transfer and its related circuits, Microwave and Millimeter-wave electronic circuits and systems design as well as Telecommunications Management. 